\title{
Does aerobic exercise effect pain sensitisation in individuals with musculoskeletal pain? A systematic review
}

\author{
Lynn Tan ${ }^{1}$, Flavia M Cicuttini ${ }^{1}$, Jessica Fairley ${ }^{1}$ Lorena Romero ${ }^{2}$, Mahnuma Estee ${ }^{1}$, Sultana Monira Hussain ${ }^{1}$ and \\ Donna M Urquhart ${ }^{1 *}$
}

\begin{abstract}
Background: Pain sensitisation plays a major role in musculoskeletal pain. However, effective treatments are limited, and although there is growing evidence that exercise may improve pain sensitisation, the amount and type of exercise remains unclear. This systematic review examines the evidence for an effect of aerobic exercise on pain sensitisation in musculoskeletal conditions.
\end{abstract}

Methods: Systematic searches of six electronic databases were conducted. Studies were included if they examined the relationship between aerobic physical activity and pain sensitisation in individuals with chronic musculoskeletal pain, but excluding specific patient subgroups such as fibromyalgia. Risk of bias was assessed using Cochrane methods and a qualitative analysis was conducted.

Results: Eleven studies (seven repeated measures studies and four clinical trials) of 590 participants were included. Eight studies had low to moderate risk of bias. All 11 studies found that aerobic exercise increased pressure pain thresholds or decreased pain ratings in those with musculoskeletal pain [median (minimum, maximum) improvement in pain sensitisation: $10.6 \%(2.2 \%, 24.1 \%)]$. In these studies, the aerobic exercise involved walking or cycling, performed at a submaximal intensity but with incremental increases, for a 4-60 min duration. Improvement in pain sensitisation occurred after one session in the observational studies and after 2-12 weeks in the clinical trials.

Conclusions: These findings provide evidence that aerobic exercise reduces pain sensitisation in individuals with musculoskeletal pain. Further work is needed to determine whether this translates to improved patient outcomes, including reduced disability and greater quality of life.

Keywords: Aerobic exercise, Pain sensitisation, Musculoskeletal pain, Systematic review, Pressure pain threshold

\section{Background}

Musculoskeletal pain is a leading global health problem. One in three people worldwide live with a musculoskeletal condition, which is characterised by pain and disability, leads to reduced quality of life, and results

\footnotetext{
*Correspondence: Donna.Urquhart@monash.edu

${ }^{1}$ Department of Epidemiology and Preventive Medicine, School of Public

Health and Preventive Medicine, Monash University, 553 St Kilda Rd, Melbourne, Victoria 3004, Australia

Full list of author information is available at the end of the article
}

in a huge economic burden [1-3]. While chronic musculoskeletal pain includes a heterogeneous group of conditions, which are characterised by pain in different regions/joints and with varying pathogeneses [4], pain sensitisation has been identified as a significant component of these conditions [5]. Pain sensitisation, according to the International Association for the Study of Pain, is defined as "an increased responsiveness of nociceptive neurons to their normal input, and/ or recruitment of a response to normally subthreshold original author(s) and the source, provide a link to the Creative Commons licence, and indicate if changes were made. The images or other third party material in this article are included in the article's Creative Commons licence, unless indicated otherwise in a credit line to the material. If material is not included in the article's Creative Commons licence and your intended use is not permitted by statutory regulation or exceeds the permitted use, you will need to obtain permission directly from the copyright holder. To view a copy of this licence, visit http://creativecommons.org/licenses/by/4.0/. The Creative Commons Public Domain Dedication waiver (http://creativeco mmons.org/publicdomain/zero/1.0/) applies to the data made available in this article, unless otherwise stated in a credit line to the data. 
inputs" [6]. Pain sensitisation is common in musculoskeletal pain cohorts, with reports of $30 \%$ of individuals with osteoarthritis (OA) [7], and 66\% of low back pain patients exhibiting high pain sensitivity [8]. Moreover, there is growing evidence highlighting the association between pain sensitisation and significant disability and poor quality of life in musculoskeletal conditions $[9,10]$. Currently therapies targeting pain sensitisation are limited [11, 12], with unclear evidence for the efficacy of commonly used interventions, such as off label (unapproved indications) tricyclic antidepressants, as well as the major issue of significant side effects associated with opioid use, including addiction, fatality and hyperalgesia [13-17].

Exercise is recommended for the management of musculoskeletal pain in national and international treatment guidelines [18-21]. While there are many types of exercise, including aerobic, strengthening, and stretching, there are limited data available to indicate which type of exercise and dosage is effective for reducing pain sensitisation. Aerobic exercise, which typically includes walking, stationary cycling, or stepping, involves physical exercise of low to high intensity that depends primarily on the aerobic energy-generating process, i.e. the use of oxygen to meet energy demands [22, 23]. It has been proposed that aerobic exercise may reduce pain sensitisation by activating descending pain inhibitory mechanisms and/or endogenous opioid and cannabinoid systems in individuals with musculoskeletal pain [24-26].

Narrative reviews and a meta-analysis have been conducted to determine whether aerobic exercise effects pain sensitivity in both healthy individuals and those with chronic pain [22, 27-30]. A meta-analysis by Naugle et al. (2012) found that in healthy individuals a bout of aerobic exercise resulted in a reduction in sensitivity to painful stimuli, termed as 'exercise-induced hypoalgesia'. However, the review also included studies examining chronic pain and found conflicting findings, with aerobic exercise producing both a hyper and hypoalgesic response [27]. While these contrasting responses may be explained by half of the included studies examining the response of individuals with fibromyalgia [31, 32] and chronic fatigue syndrome [33] and finding a hyperalgesic effect, the role of aerobic exercise in chronic musculoskeletal conditions, such as low back and knee pain, is still unclear. Thus, the research questions to be examined in this systematic review were:

1. Does aerobic exercise effect pain sensitisation in individuals with musculoskeletal pain? If so, what effect does it have?

2. What type and dosage of aerobic exercise is associated with a change in pain sensitisation?

\section{Methods}

This systematic review was conducted according to the 2020 PRISMA guidelines [34].

\section{Search strategy}

We performed electronic searches of six databases, including OVID MEDLINE, OVID Embase, OVID EBM Reviews Cochrane Central Register of Controlled Trials, OVID PsycINFO, CINAHL and SPORTDiscus, from database inception to 26th March 2021. An initial search for studies was conducted in OVID Medline, and an analysis of text words and subject terms was then used to develop the search strategy, with subject classification systems investigated for each database. The final searches of all six databases, covering the key concepts of aerobic physical activity, pain sensitisation and musculoskeletal pain, were performed using the appropriate specifications for each database. The comprehensive search strategy for OVID Medline is shown in the Supplementary File 1 . The searches were limited to studies of adults $(\geq 18$ years of age) and those published in the English language. We also searched the reference lists of systematic and narrative reviews and meta-analyses in the field, as well as the studies included in this review.

\section{Study identification}

Titles and abstracts were assessed by two authors (LT, DU) for relevance using Covidence and the full texts were retrieved for relevant studies. We included studies that examined the role of aerobic exercise on pain sensitisation in musculoskeletal pain cohorts (Table 1). Aerobic exercise was defined as physical activity, such as walking, cycling and stepping, which involves large muscle groups and the consumption of oxygen to

Table 1 Inclusion criteria

Design
- Observational studies
- Randomised or quasi-randomised trials
- English language studies
Participants
- Individuals with musculoskeletal pain, but not specific subgroups, such
as fibromyalgia, chronic fatigue syndrome, and whiplash, that have been
shown to differ in their response to pain.
Intervention
- Aerobic physical activity
Comparisons
- Aerobic physical activity versus control
- Aerobic physical activity and intervention A versus intervention A
Outcome measures
- Pain sensitisation, including pressure or thermal pain thresholds or pain
ratings


generate energy $[22,23]$. Pain sensitisation was measured using validated, quantitative measures, such as pressure and thermal pain thresholds (PPT/TPT), where the minimum mechanical force or heat applied that induces pain is determined, and pressure and thermal pain ratings, where perceived pain is recorded on a pain rating instrument (e.g. visual analogue scale) during the pressure or thermal stimulus respectively [3537]. Musculoskeletal pain was defined as pain caused by varying pathogeneses in muscles, bones, joints and associated tissues such as tendons and ligaments [38]. We excluded studies that examined the following exercise types; eccentric, strengthening, resistance, weight training, stabilization, postural correction, stretching and/or mobility, and neuromobilisation. Studies that examined Yoga, Tai Chi, Jyoti, Qigong, Pilates, and Zumba and did not specify an aerobic nature were also excluded, as were studies that assessed the effect of aerobic exercise on pain intensity, rather than pain sensitivity.

While we included clinical trials that examined aerobic exercise combined with another intervention compared to that intervention alone, we excluded trials that examined the effectiveness of a combination of treatments or compared aerobic exercise with another intervention. Specifically, we excluded studies that examined the following conditions; complex regional pain syndrome, fibromyalgia, rheumatoid arthritis, chronic fatigue syndrome, headache, migraine, temporomandibular joint disorders, myofascial pain, whiplash and inflammatory arthritis.

\section{Data extraction}

Two authors (LT and DU) extracted and tabulated information on the characteristics of the included studies, including; demographics of the cohort, type of musculoskeletal pain, study inclusion and exclusion criteria, measurement of pain sensitisation, types and dosage of the aerobic exercise, and the results and conclusions of the studies. PPT was defined as the minimum force applied which induces pain and thermal pain threshold was defined as the minimal heat which produces pain, signifying a quantitative value related to the mechanical sensitivity to pain of deep structures $[35,36]$. These validated measures have widespread use in evaluating muscular and joint tenderness associated with musculoskeletal conditions, and for the diagnosis and efficacy analysis of management strategies [39, 40]. An increase in pressure or thermal pain thresholds or decrease in pressure or thermal pain ratings is considered to signify hypoalgesia and a reduction in pain sensitisation $[37,41]$.

\section{Risk of bias}

Risk of bias was assessed using methods adapted from the Cochrane Collaboration for observational studies and randomised controlled trials $[42,43]$. The assessment was performed by two reviewers (JF, LT) independently and where consensus could not be achieved, a judgement was made by DU and SMH. We assessed the internal and external validity of the observational studies based on seven items. Each item was assessed as "yes", "no", "unclear" or "not applicable" and these contributed to an overall assessment of the risk of bias for each study. The overall assessment for the study was 'low' if the answer was "yes" to all items, 'moderate' if the answer was "no" or "unclear" to one or two items, or 'high' if the answer was "no" or "unclear" to more than two items. To assess the risk of bias of the randomised controlled trials, eight criteria were used to assess the internal validity of the trials. The criterion were scored as "yes", "no", or "unclear". Low risk of bias was defined as fulfilling six or more of the eight quality criteria (i.e. obtaining a 'yes' score to at least six criteria).

\section{Clinical heterogeneity}

We assessed the clinical heterogeneity of the trials in this review using the Clinical Diversity In Meta-analyses (CDIM), a new tool for assessing clinical diversity between trials in systematic reviews and meta-analyses of interventions [44]. Two authors (LT, DMU) assessed clinical diversity across the four domains of setting, population, intervention and outcome, and a total consensus score from 0 to 22 was calculated, with 0 indicating no clinical heterogeneity and 22 high levels of heterogeneity. While there is no validated tool for assessing clinical heterogeneity between observational studies, we used and adapted the same 4 domains from the CDIM to examine whether the clinical data from observational studies were homogeneous.

\section{Best evidence synthesis}

A best evidence synthesis was used to summarise the data, as it was not possible to perform a meta-analysis due to the clinical heterogeneity between the studies. The studies were ranked according to their design, with clinical trials considered to be a higher level of evidence than observational studies. Both the study design and risk of bias scores were considered when determining the evidence available.

\section{Results}

Flow of the studies through the review

Electronic searches identified 13,190 studies, 2,548 from OVID MEDLINE, 4,542 from OVID Embase, 
2,280 from CINAHL, 2,128 from OVID EBM Reviews Cochrane Central Register of Controlled Trials, 441 from PsychINFO and 1,251 from SPORTDiscus from inception to 26th March 2021 (Fig. 1). After the screening of titles and abstracts, 115 full text papers were retrieved. On examining the full text, a further 104 studies were excluded as they either: (i) investigated a healthy cohort or a cohort without musculoskeletal pain $(\mathrm{n}=55)$, (ii) did not have aerobic exercise as the only intervention or included several interventions without providing the data for aerobic exercise separately $(n=9)$, (iii) did not measure pain sensitisation as the outcome $(\mathrm{n}=27)$, or (iv) had an inappropriate study type (e.g. systematic review or narrative) $(\mathrm{n}=13)$. No additional studies were identified from reference checking of key narrative reviews, systematic reviews, and meta-analyses, resulting in a total of 11 studies being included in the review.

\section{Characteristics of included studies}

The characteristics of the included studies are reported in Table 2. Of the 11 included studies, seven studies had an experimental, repeated measures design [33, 37, 41, 45-48] and four were randomised controlled trials [49-52]. Five studies recruited individuals with chronic low back pain [33, 37, 47, 48, 52], three studies examined those with chronic musculoskeletal pain [33, 37, 45, 46, $51]$, two investigated individuals with neck pain $[49,50]$, and one included individuals with knee pain due to OA [41]. Four studies were conducted in Denmark [45-47, 50], two studies were undertaken in the United States of America [37, 52], and the remaining studies were conducted in Belgium [33], Ireland [41], Turkey [51], Spain [48] and Poland [49]. Three studies were published before 2010 (in 2005 [37], 2009 [50] and 2010 [33]), 5 studies were published between 2016-2018 [41, 45, 46, 49, 51], and the remaining 3 studies were published in 2020 and $2021[47,48,52]$.

\section{Characteristics of participants}

The studies ranged from having 8-96 participants. Participants had a mean age ranging from 34 to 56 years, except for the study on knee OA which did not specify

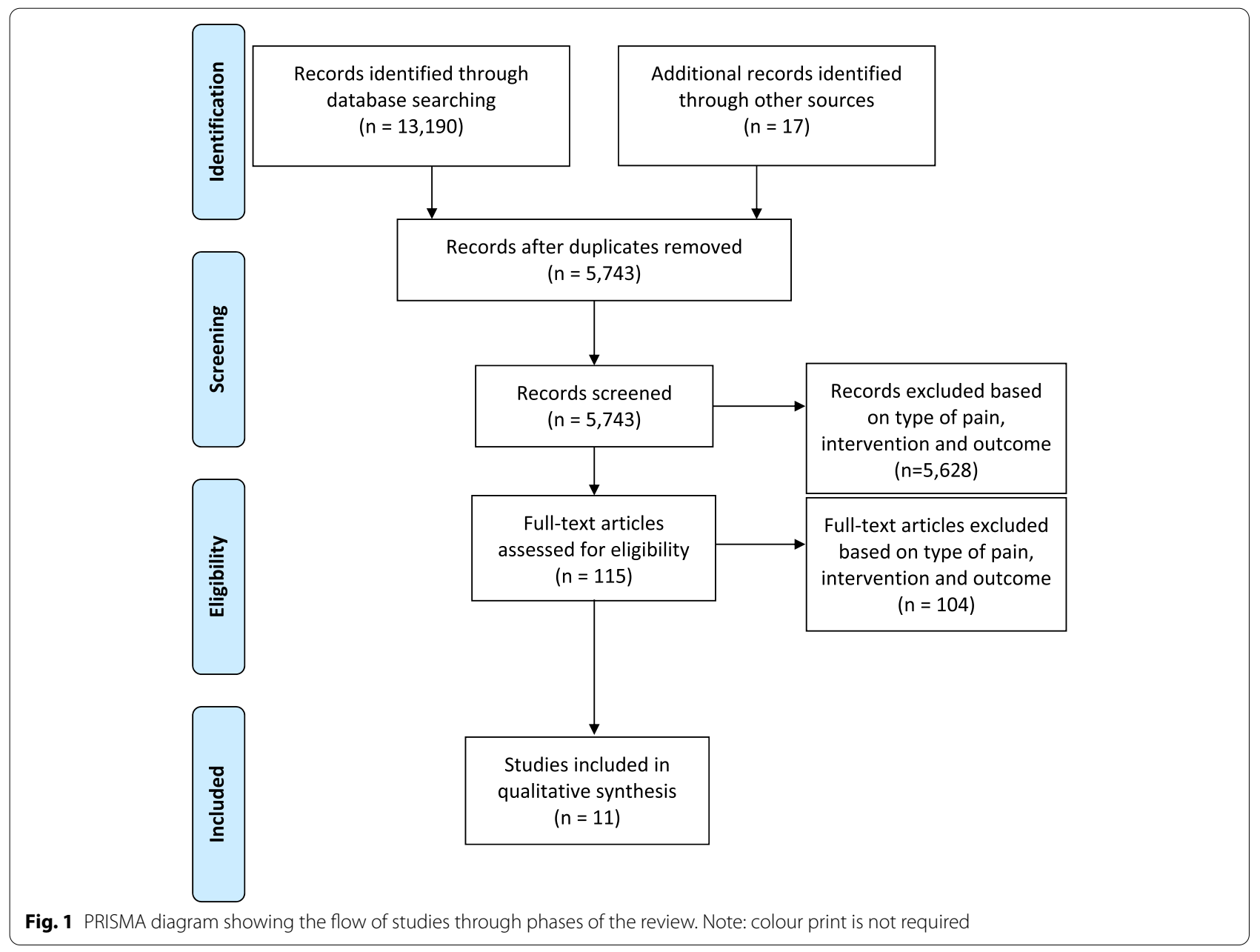




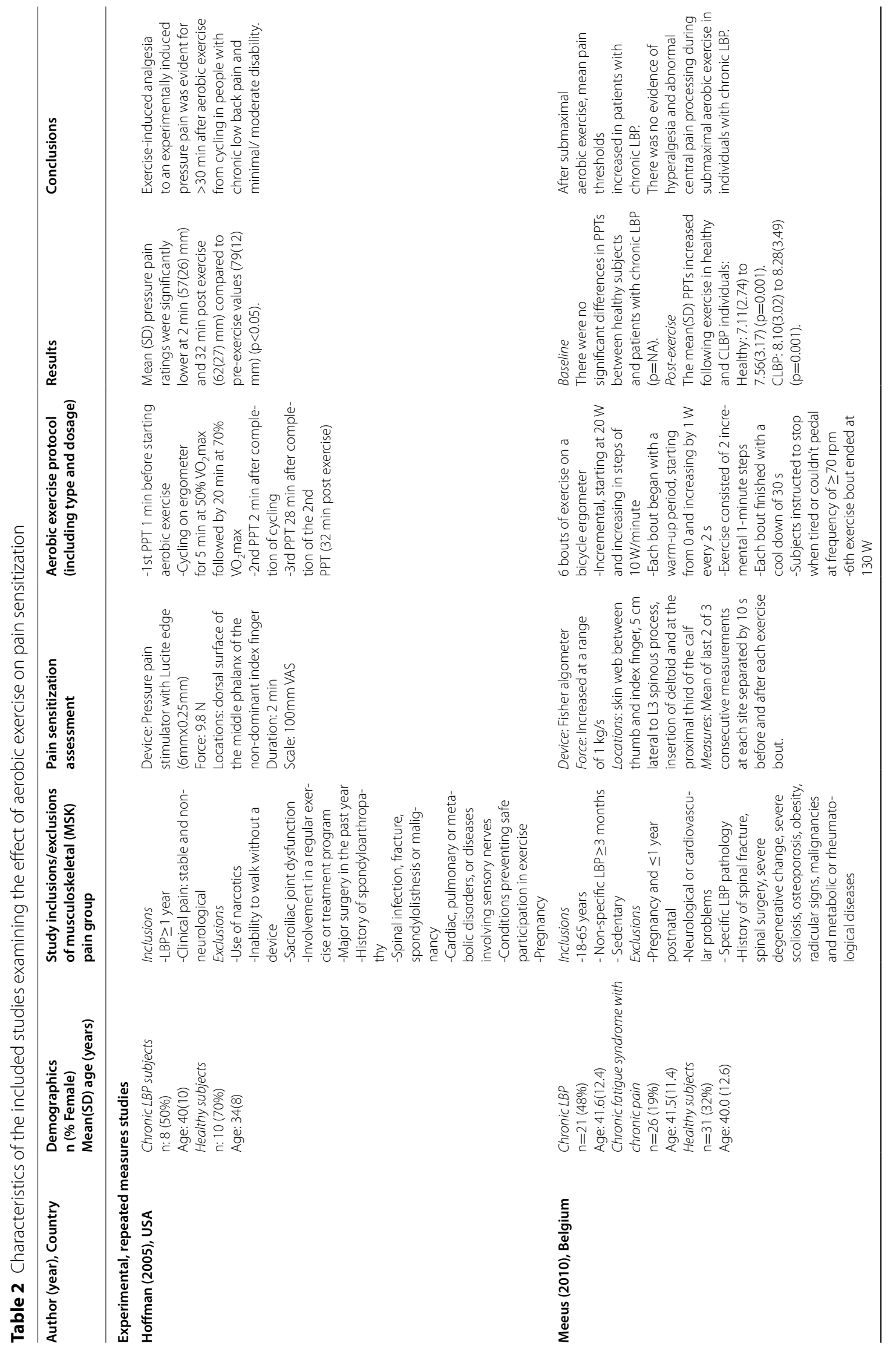




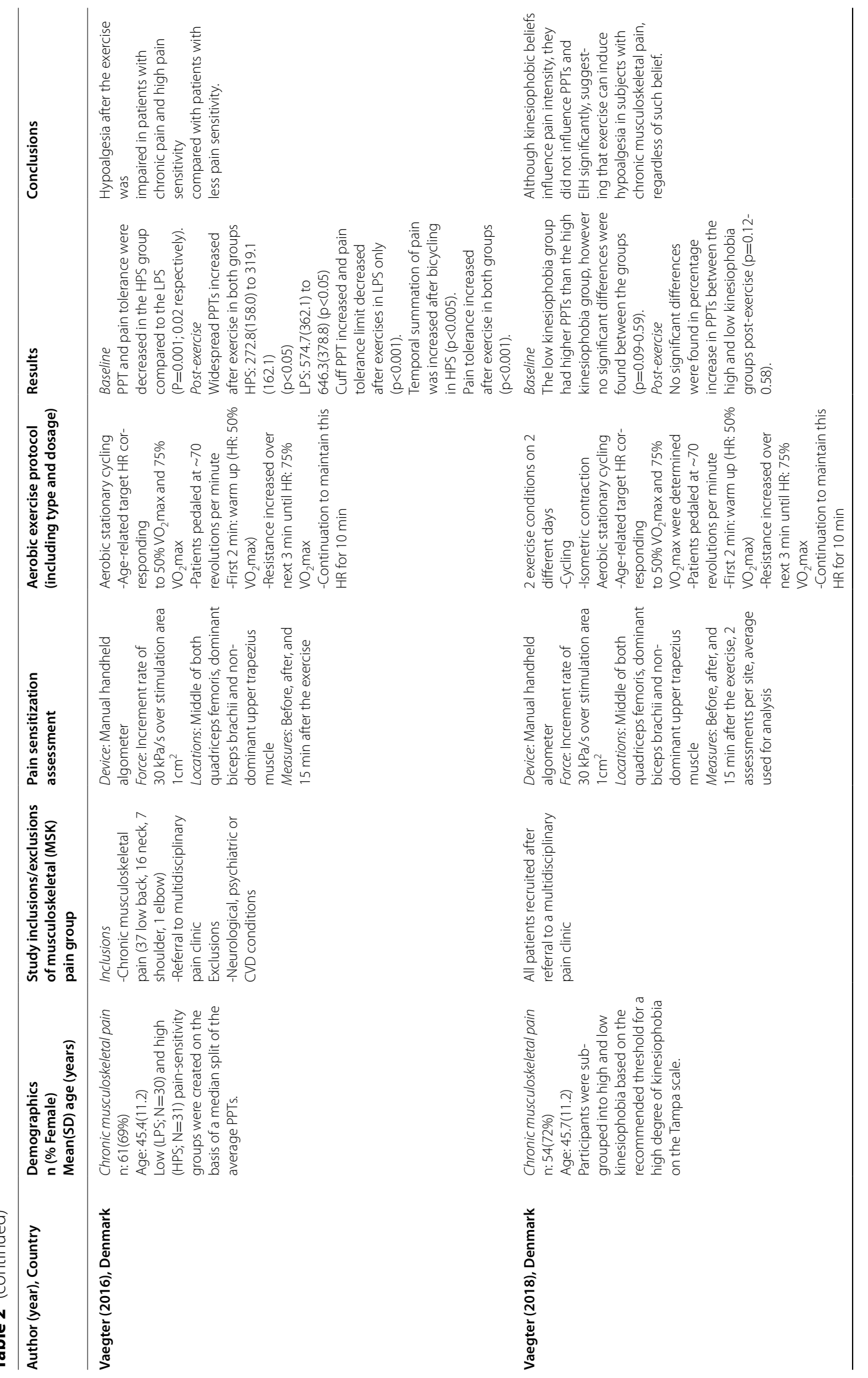




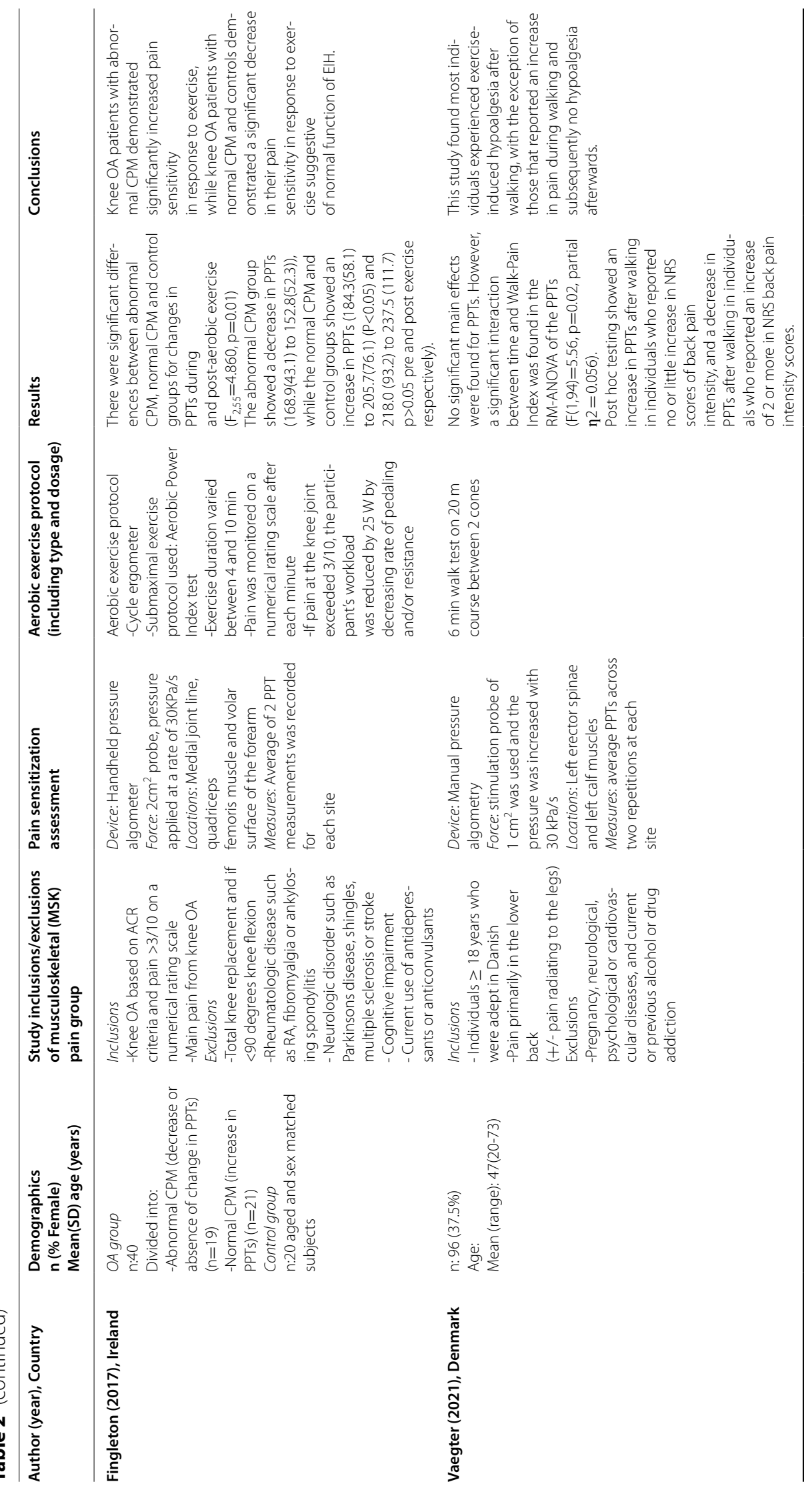




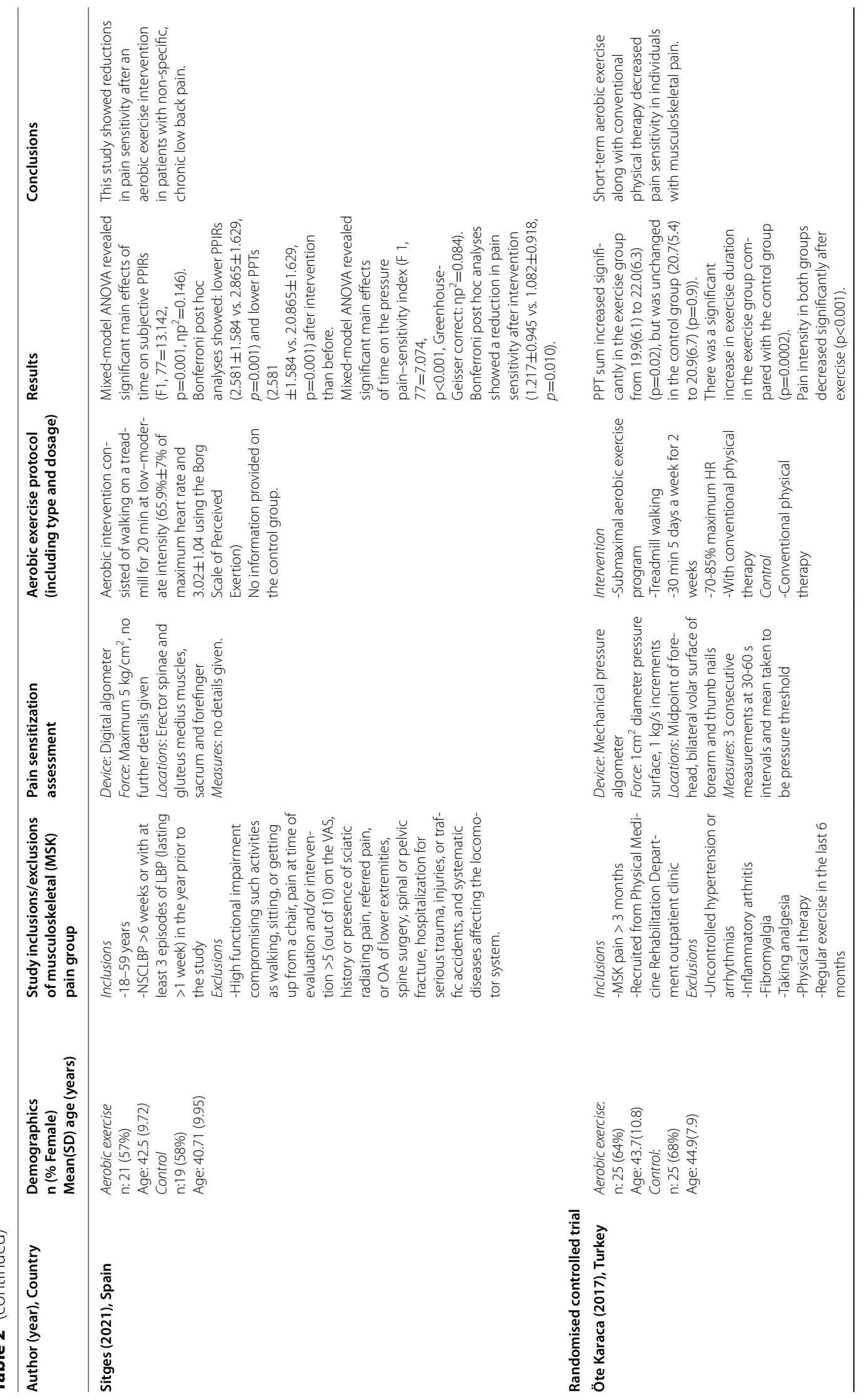




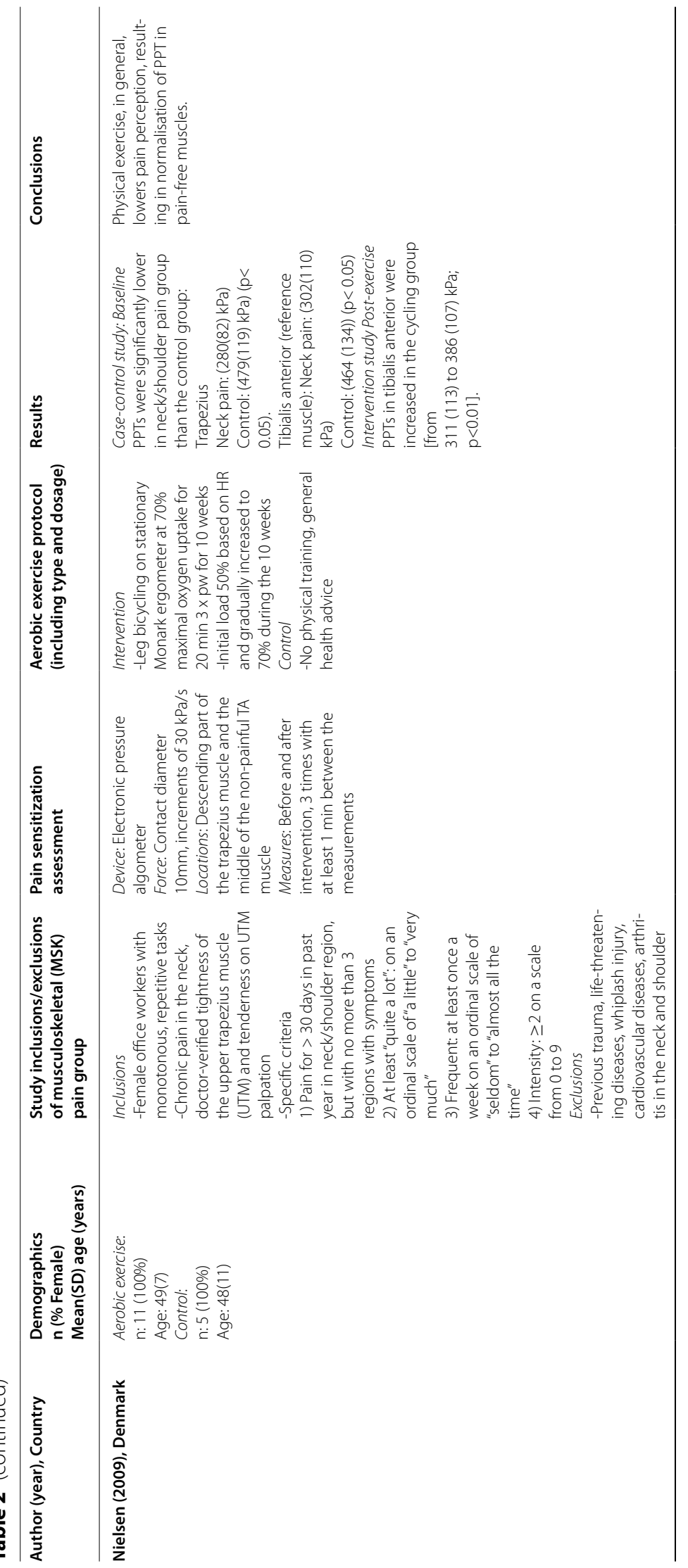




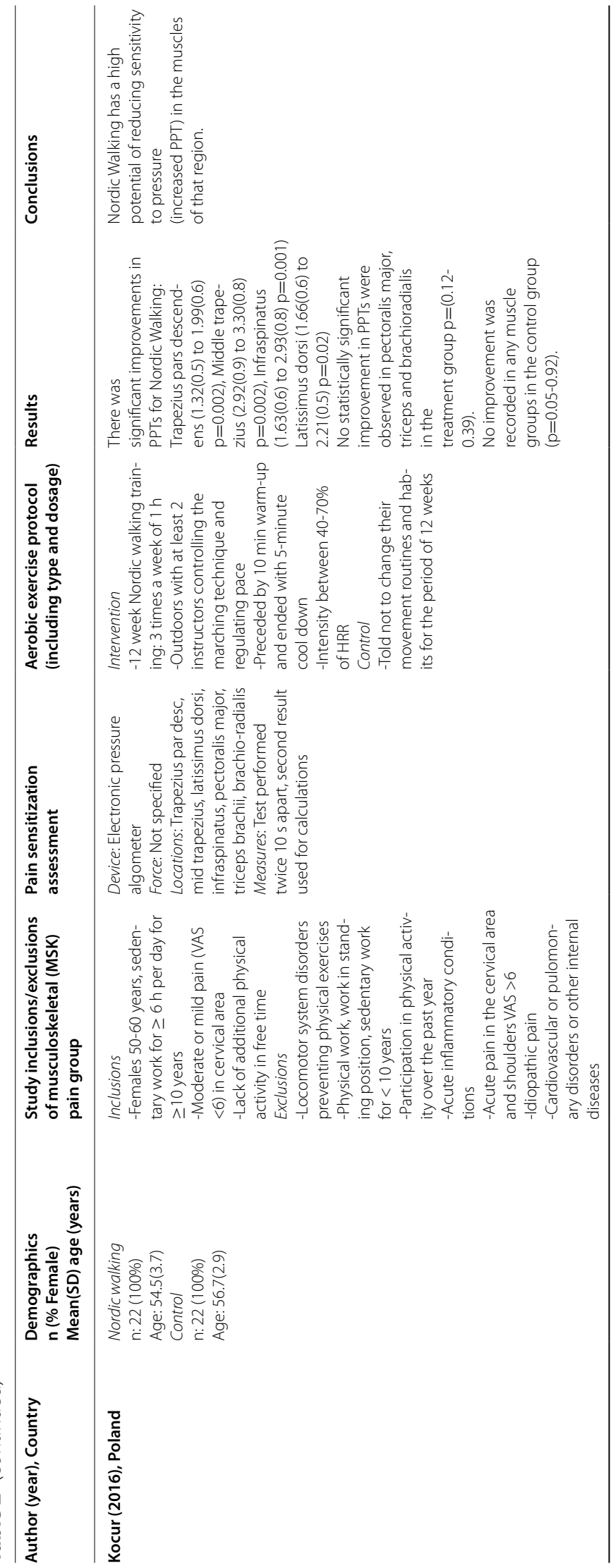




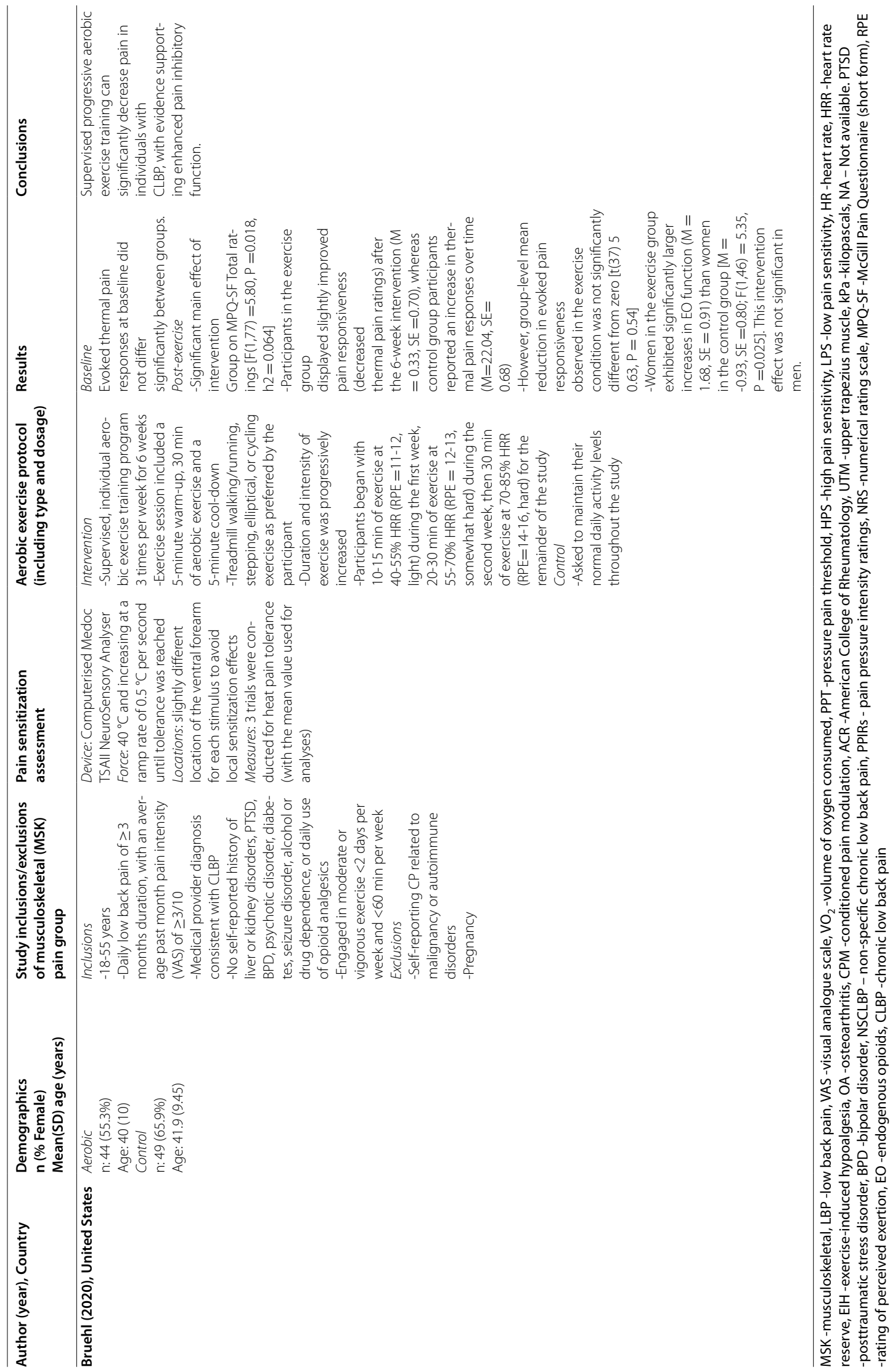


the mean age of the cohort [41]. Overall $38-100 \%$ of the participants were female. However, one study did not report the number of males and females [41].

\section{Definitions of musculoskeletal pain}

A variety of definitions of musculoskeletal pain were used in the 11 studies. Of the 5 low back studies, two studies defined low back pain as non-specific pain lasting longer than three months $[33,52]$, and the other studies used definitions such as clinically stable, non-neurological pain lasting longer than one year [37], pain in the lower back with or without pain in the lower limbs [47], and pain lasting $>6$ weeks or at least 3 episodes ( $>1$ week) in the last year [48]. In the 2 studies of chronic musculoskeletal pain, more than half of the cohort experienced low back pain, and the remainder reported neck, shoulder and elbow pain, with the duration of pain not specified $[45,46]$. Ote Karaca et al. recruited individuals who had regional musculoskeletal pain exceeding three months in duration [51]. Neilsen et al. recruited participants who had neck or shoulder pain lasting for more than 30 days [50], while Kocur et al. recruited participants who had cervical pain with the exclusion of "acute pain in the cervical area and shoulders" [49]. In the study by Fingelton et al. which examined individuals with knee OA, OA was diagnosed based on the American College of Rheumatology classification, and needed to be the main source of pain, and exceed 3 on an 11 point numerical rating scale [41].

Four of the included studies classified participants into subgroups based on pain sensitivity, kinesiophobia, selfreported pain intensity during activity, and conditioned pain modulation [41, 45-47]. Vaegter et al. (2016) examined high and low pain sensitivity subgroupsbased on a median split of the average PPTs for men and women [45], while their later study in 2018 examined high and low kinesiophobia subgroups, where the subgroups were created based on the recommended threshold for a high degree of kinesiophobia using the Tampa Scale of Kinesiophobia (score $\geq 38$ ) [46]. In addition, the most recent study by Vaegter et al. examined change in pain sensitisation in individuals that reported an increase $(\geq 2 / 10)$ in their back pain during walking compared to those that reported no or limited increase $(<2 / 10)$ [47]. The study of OA classified participants as "abnormal conditioned pain modulation (CPM)" where there was a decrease in PPTs or absence of change, or "normal CPM" where there was an increase in PPTs [41].

\section{Aerobic exercise prescription}

Five experimental, repeated measures studies and one of the randomised controlled trials used cycle ergometry as the prescribed aerobic exercise [33, 37, 41, 45, 46,
50], while one repeated measures study and randomised controlled trial used treadmill walking $[48,51]$ and the remaining 3 studies used Nordic walking [49], a 6 min walk test [47], and selection from a range of aerobic activities, including walking, stepping, elliptical exercise and cycling, as per the participant's preference $[49,52]$.

The degree of exertion was standardised in two ways. The first involved calculating target $\mathrm{VO}_{2}$ maximums through maximal or submaximal exercise tests prior to the prescribed aerobic exercise, while the second was based on target age-related heart rates [37, 41, 45, 46, 4852]. Two studies did not standardise the degree of exertion $[33,47]$.

In the 7 repeated measures studies, the exercise protocols were submaximal, incremental and completed in a single setting over 4 to $30 \mathrm{~min}$, ranging from: (i) commencing at $50 \%$ maximal $\mathrm{VO}_{2}$ for $5 \mathrm{~min}$ and increasing to $70 \%$ maximal $\mathrm{VO}_{2}$ for $20 \mathrm{~min}$ as a single bout [37], (ii) starting at $20 \mathrm{~W}$ after a short warm-up period and increasing by $10 \mathrm{~W}$ per minute for 2 min per exercise bout followed by a $30 \mathrm{~s}$ cooling down period for 6 exercise bouts [33], (iii) starting at a heart rate corresponding to $50 \%$ maximal $\mathrm{VO}_{2}$ for $2 \mathrm{~min}$, increasing to $75 \%$ over $3 \mathrm{~min}$ and continuation at that heart rate for a further $10 \mathrm{~min}$ in a single bout $[45,46]$, (iv) starting at $25 \mathrm{~W}$ and increasing by $25 \mathrm{~W}$ per minute to $75 \%$ age-predicted maximal heart rate for a duration of 4-10 min [41] and (v) increasing intensity to a low-moderate intensity of $70 \%$ of maximum heart rate [48]. One study did not have a specific exercise protocol but instructed participants to walk as far as possible within the 6 min time frame [47].

The randomised controlled trials described a more prolonged submaximal aerobic exercise protocol. Ote Karaca et al. described treadmill-based aerobic exercise at an intensity of $70-85 \%$ age-related target heart rate for $30 \mathrm{~min}$ five days a week for two weeks [51]. Neilsen et al. described a stationary, ergometer-based aerobic exercise at $50 \%$ initial load based on heart rate, gradually increasing to $70 \%$ during the 10 weeks [50]. Kocur et al. described Nordic walking between 40 and $70 \%$ heart rate reserve for $1 \mathrm{~h}, 3$ times a week for 12 weeks with each session preceded by a warm up and followed by a cool down phase [49]. Bruehl et al. started participants with 10-15 min of exercise at 40-55\% heart rate reserve during the first week, followed by $20-30 \mathrm{~min}$ at a $55-70 \%$ heart rate reserve during the second week, and then $30 \mathrm{~min}$ at a $70-85 \%$ heart rate reserve for the remainder of the study [52].

\section{Assessment of pain sensitisation}

The outcome we assessed was pain sensitisation, which was measured with pressure pain testing in 10 studies and thermal pain testing in one study. Pressure pain 
testing involved producing pressure over a small surface area, participants identifying when a pressure sensation was first perceived as pain and the corresponding pressure or the pain level being recorded. Sites of pressure pain testing were variable $[33,37]$, with the quadriceps femoris $[41,45,46]$, and $[45,46,51]$ trapezius $[45,46$, $49,50]$, most commonly used [49]. In most of the studies, pressure applied was incremented either at a rate of $1 \mathrm{~kg} / \mathrm{s}$ or $30 \mathrm{kPa} / \mathrm{s}$ over $1 \mathrm{~cm}^{2}$ or $2 \mathrm{~cm}^{2}$ [33, 37, 41, 45, 46, 49-51]. In contrast, the trial by Bruehl performed thermal pain testing which involved applying a thermode to the skin of the forearm starting at $40{ }^{\circ} \mathrm{C}$ and increasing at a ramp rate of $0.5{ }^{\circ} \mathrm{C}$ per second until tolerance was reached, at which the temperature was recorded and the participant rated their pain intensity [52].

\section{Risk of bias}

The studies in this review were largely of low to moderate risk of bias, with two studies having low risk of bias [45, 46], six studies having moderate bias [33, 37, 41, 47-49], and three studies having high risk of bias [50-52]. All seven observational studies adequately assessed both the exposures and outcomes and ensured that the co-interventions were similar between both cohorts. However, four studies did not match exposed and unexposed for all variables that are associated with the outcome of interest or adjust for these prognostic variables [33, 37, 47, 48], and 3 of the studies did not draw cohorts from the same population [33, 37, 41]. One of the 4 randomised controlled trials reflected a moderate risk of bias [49], while the other three reflected a high risk of bias as they had no allocation concealment and did not mention blinding of participants or personnel [50-52].

\section{Clinical heterogeneity}

Based on the Clinical Diversity In Meta-analyses (CDIM) tool[44], we calculated a score of 17 (of a total score of 22) for the clinical trials included in this review, indicating high clinical heterogeneity. In particular, scores of 2 were evident in 3 of the 4 domains, including the population, intervention and outcome domains, reflecting significant heterogeneity. Similarly, when examining the observational studies we found significant clinical heterogeneity, which was evident across all domains.

\section{Effect of aerobic exercise on pain sensitisation Question 1: Does aerobic exercise effect pain sensitisation? If so, what effect does it have?}

All 11 studies reported a hypoalgesic effect of aerobic exercise on pain sensitisation in participants with chronic musculoskeletal pain. A summary of these results is presented in Tables 2 and 3. Moreover, when we examined the risk of bias of the included studies and only considered the 8 studies that had low to moderate risk of bias, our findings of a reduction in pain sensitisation following aerobic exercise, remained unchanged. However, there was evidence from two studies that examined specific musculoskeletal pain subgroups, including those with abnormal conditioned pain modulation or an increase in low back or leg pain while walking, that not all subgroups experienced a reduction in pain sensitisation.

The five studies investigating low back pain reported an improvement in pain sensitisation after performing aerobic exercise. The study by Hoffman et al. reported a statistically significant decrease in pressure pain at two minutes and $32 \mathrm{~min}$ after aerobic exercise on a cycle ergometer [37]. Meeus et al. reported a similar result with an increase in mean PPTs immediately following aerobic exercise [33], as did the study by Sitges et al. which reported lower pain sensitivity and pressure painintensity ratings following $20 \mathrm{~min}$ of treadmill walking [48]. The study by Vaegter et al. reported an increase in PPTs after walking in individuals who reported no or little increase in pain while walking [47], while the clinical trial by Bruehl et al. reported larger reductions in evoked pain responsiveness in those who undertook a 6 week program involving their preferred aerobic exercise compared to controls [52].

The three studies of chronic musculoskeletal pain, including the randomised controlled trial and the two repeated measures studies, reported a statistically significant increase in pain tolerance after aerobic exercise [45, $46,51]$.

The two studies examining chronic cervical pain reported increased PPTs at specific sites, although they did not report an overall mean or summation score [49, 50]. Neilsen et al. reported increased PPTs at the tibialis anterior site without significant increase in the descending trapezius, the other site measured [50]. Kocur et al. reported significant increases in PPTs in 4 of 7 of the sites measured [49]. In the control groups of both of these studies, no increases in PPTs at any of the sites was observed $[49,50]$.

In the study by Fingleton et al., mean PPTs were significantly increased post exercise among participants with knee OA with a normal conditioned pain modulation, but not among participants with abnormal conditioned pain modulation [41]. Vaegter et al. found increased widespread PPTs in both high and low pain sensitivity groups with effect sizes of 0.77 and 0.52 respectively, but concluded that exercise-induced hypoalgesia was slightly impaired in patients with high pain sensitivity compared with patients with low sensitivity [45].

Of the 11 included studies, 7 studies reported mean PPTs and 2 studies reported mean pain ratings pre and post exercise allowing the percentage improvement in 
pain sensitisation following aerobic exercise to be determined (Table 3) [33, 41, 45, 46, 48, 50, 51]. Two studies did not report mean pre and post PPTs or pain ratings. Based on the mean change in PPTs, we calculated a median (minimum, maximum) percentage improvement in pain sensitisation of $10.6 \%(2.2 \%, 24.1 \%)$.

\section{Question 2: What type and dosage of exercise, including duration, intensity and frequency of exercise, was required to achieve an effect?}

All of the 11 included studies reported a hypoalgesic effect when they examined aerobic exercise that involved:

1) Walking, cycling or the participants' preferred aerobic exercise (6 studies examined cycling [33, 37, 41, $45,46,50], 4$ examined walking $[47-49,51]$ and one study investigated treadmill walking/running, stepping, elliptical, or cycling [52]).

2) Submaximal exercise (9 of the 11 studies were objectively submaximal based on percentage $\mathrm{VO}_{2}$ or target $\mathrm{HR}\left(50-75 \% \mathrm{VO}_{2},[37,45,46], 40-85 \%\right.$ heart rate reserve $[49,52]$, or $66-85 \%$ maximal heart rate $[33$, 41, 47-52].

3) exercise of 4-60 min in duration (with 2 studies having varying exercise times depending on participants' tolerance $[33,41])$,

4) a single bout (7 of the 11 studies) [33, 37, 41, 45-48], with 4 studies examining interventions up to 5 times a week and up to 12 weeks [49-52].

5) mostly incremental (at least 7 of the 11 studies) [33, $37,41,45,46,50,52]$.

It would therefore seem that a single session of submaximal, incremental exercise involving walking or cycling of at least $4 \mathrm{~min}$ duration based on $50-75 \% \mathrm{VO}_{2}$, $40-85 \%$ heart rate reserve $[49,52]$, or $66-85 \%$ maximal heart rate $[41,48,50,51]$ may achieve a hypoalgesic effect immediately post exercise.

\section{Was there an improvement in PPTs over both shorter and longer time periods?}

While the majority of the repeated measures studies examined pain sensitisation before and immediately after aerobic exercise, two studies measured pain sensitisation a period of time after exercise [37, 45]. Hoffman et al. reported pressure pain ratings that continued to be lower compared with pre-exercise at $32 \mathrm{~min}$ post-exercise [37], while Vaegter et al. reported PPTs that were no different with pre-exercise at $15 \mathrm{~min}$ post-exercise [45, 46] The study by Hoffman et al. that had a hypoalgesic effect at $32 \mathrm{~min}$ post exercise prescribed aerobic exercise for a 25 min period, while the study by Vaegter et al. that lost the hypoalgesic effect at $15 \mathrm{~min}$ post exercise had a 15 min aerobic exercise prescription $[37,45]$

In contrast to the repeated measures studies, the four randomised controlled trials assessed pain sensitisation after 2, 6, 10 and 12 weeks of an aerobic exercise program. Ote Karaca et al. reported a statistically significant increase in sum of PPTs after a 2 week intervention (with 5 of 7 components of the sum having individually statistically significant increases) [51]. Neilsen et al. reported increased PPTs at one of two tested sites (tibialis anterior site but not the trapezius) after 10 weeks of aerobic exercise, and Kocur et al. reported increased PPTs in four of the seven sites after 12 weeks of aerobic exercise. However, these 2 studies did not report a mean or comparison of the widespread or collective PPTs across the sites tested $[49,50]$. Moreover, Bruehl et al. reported an decrease in pain sensitivity based on the MPQ-total pain measure in response to a thermal pain stimulus at 6 weeks following an aerobic exercise program [33, 37, 41, 45-52].

\section{Discussion}

This systematic review provides evidence to suggest that aerobic exercise reduces pain sensitisation in individuals with musculoskeletal pain. All 11 studies concluded that aerobic exercise produced a hypoalgesic effect in their musculoskeletal pain cohort, with median (minimum, maximum) improvement in pain sensitisation of $10.6 \%(2.2 \%, 24.1 \%)$ post exercise. Moreover, the studies showed that aerobic exercise, involving walking or cycling, at a submaximal intensity of $50-75 \%$ maximal $\mathrm{VO}_{2}$ or $40-85 \%$ maximal heart rate/heart rate reserve and with incremental increases, for a 4-60 min duration, and for a single session or 1-5 sessions per week for up to 12 weeks resulted in a hypoalgesic effect. However, there was evidence from studies of specific patient subgroups, including individuals with abnormal conditioned pain modulation or an increase in low back or leg pain while walking, that performing aerobic exercise may not reduce pain sensitization in all individuals with musculoskeletal pain. Given the lack of effective treatments for pain sensitisation $[11,12]$, the escalating use of off-label antidepressants $[53,54]$, and the alarming use and side-effects associated with opioids [13-17], these findings highlight the need for further investigation to determine whether aerobic exercise may be a safe and effective, non-pharmacological "pill" for the treatment of pain sensitisation in individuals with musculoskeletal pain.

Of the 11 studies included in this review, all reported that aerobic exercise resulted in hypoalgesia in individuals with musculoskeletal pain. This has not previously been shown. A 2012 meta-analysis by Naugle et al [27] investigated the effects of aerobic exercise on 


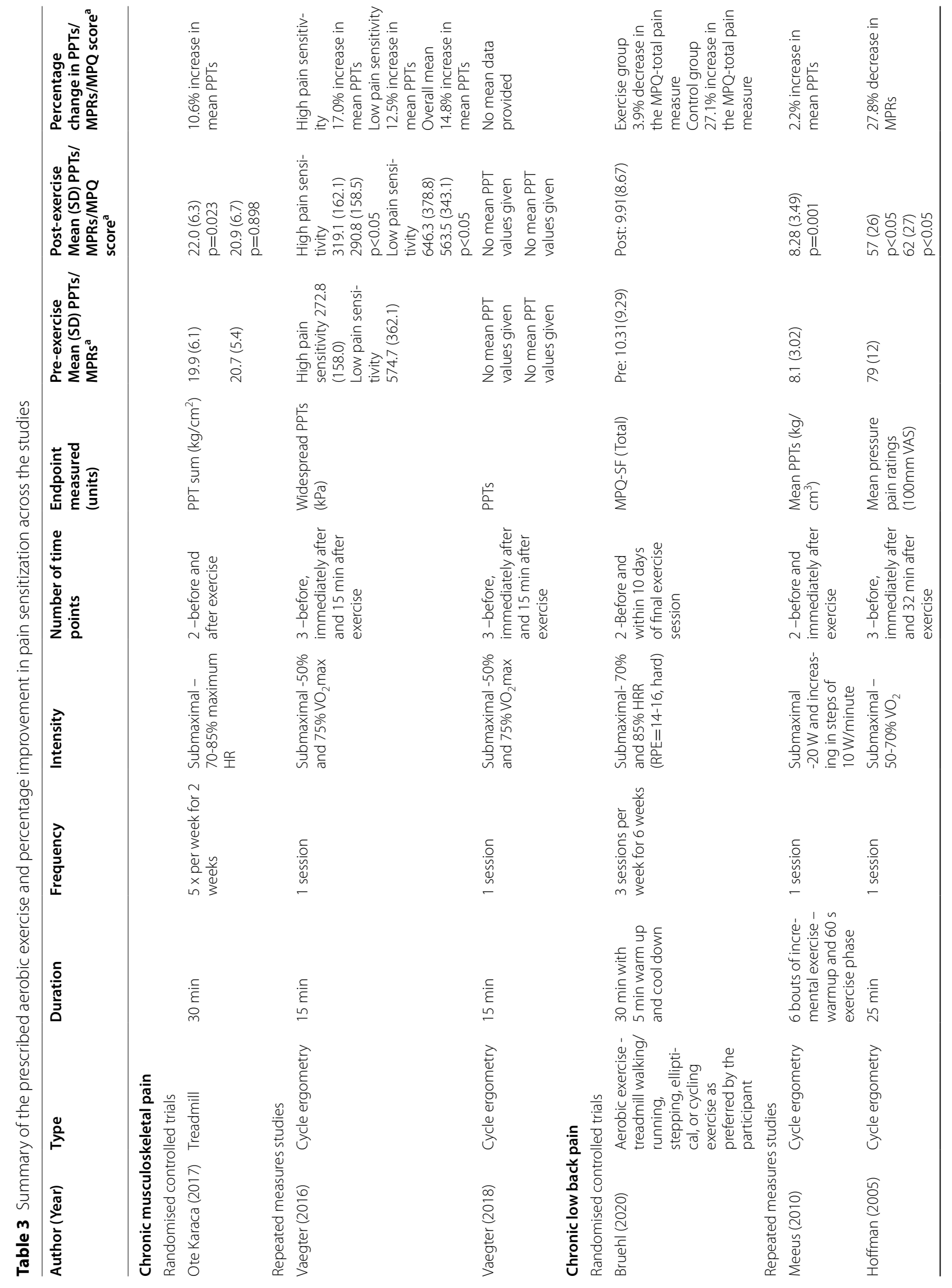




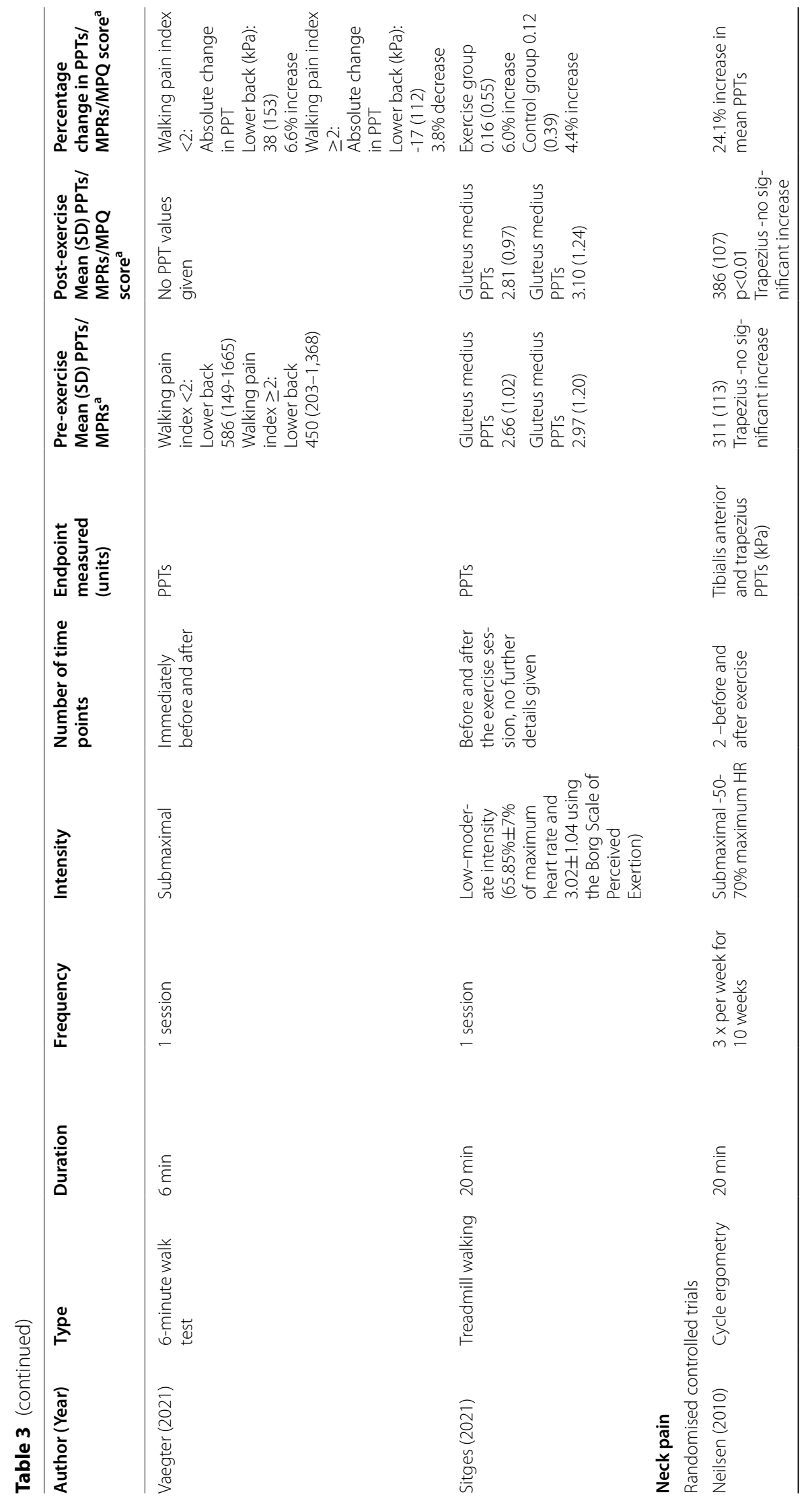




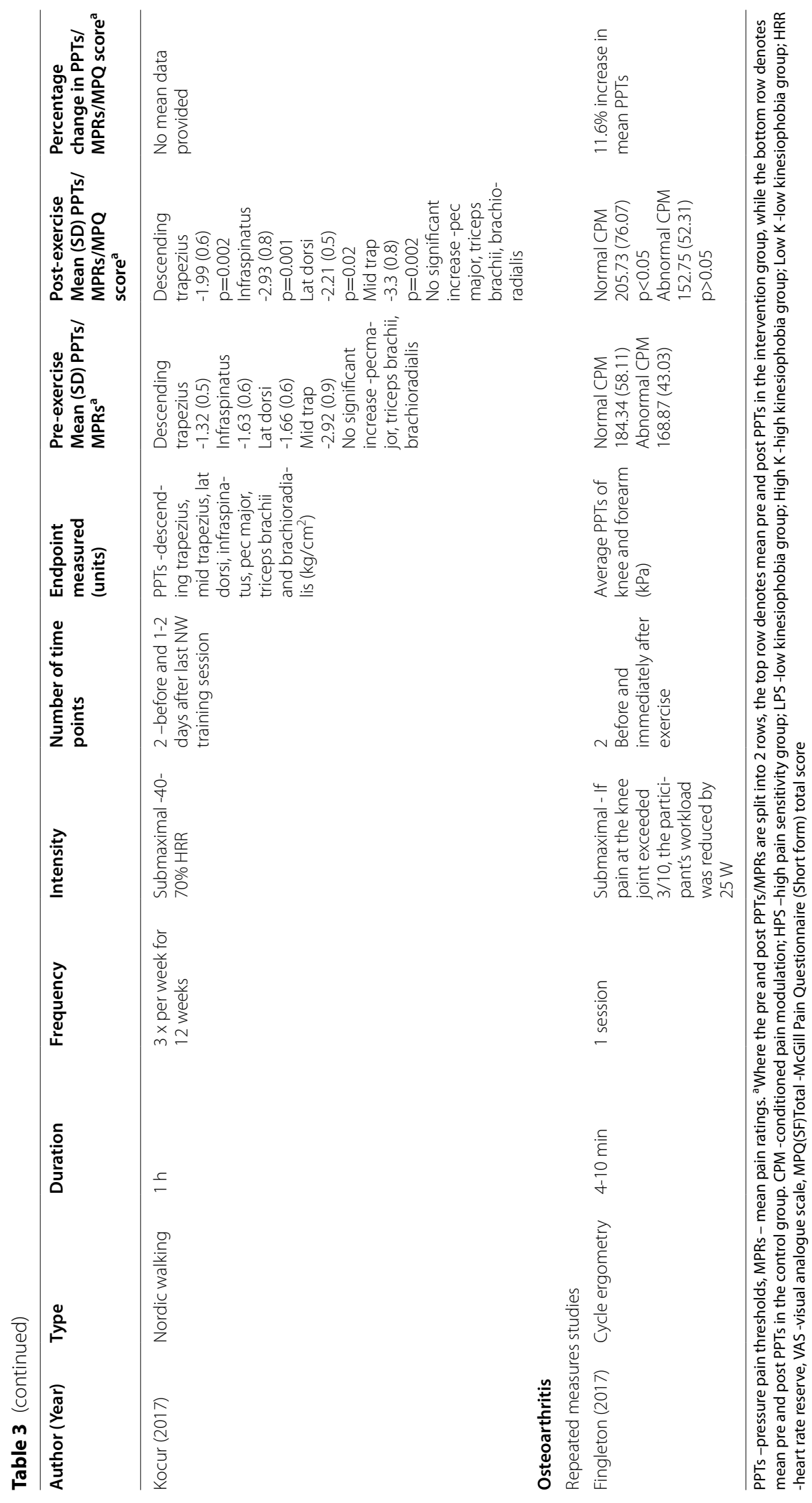


pain sensitisation in both healthy adults and those with chronic pain. They concluded that there was a trend for a beneficial effect for individuals with chronic pain, but the results were highly variable, particularly with regards to the magnitude and direction of the effect sizes [27]. It was proposed that this may be explained by 3 of the 5 studies included in the meta-analysis investigating individuals with fibromyalgia or chronic fatigue syndrome, which differed in their response to aerobic exercise and demonstrated a hyperalgesic response.

This systematic review provides preliminary data to suggest the type and dosage of aerobic exercise which might be required to achieve a reduction in pain sensitisation. Among the studies in this review, there were a range of exercise parameters utilised in relation to frequency, intensity and duration. Overall, the studies found submaximal, incremental exercise that involved walking or cycling for at least 4 min duration based on $50-75 \%$ maximal $\mathrm{VO}_{2}, 40-85 \%$ heart rate reserve, or $66-85 \%$ maximal heart rate on one or multiple occasions is needed to achieve a reduction in pain sensitisation. While the repeated measures studies prescribed a single bout of exercise, the clinical trials examined effects of 2-12 weeks of aerobic exercise and showed that there was an improvement in pain sensitisation both immediately and over periods of 2, 6, 10 and 12 weeks. Figure 2 provides a schematic representation of the effect of aerobic exercise on pain sensitisation, summarising the key features of aerobic exercise that were found to produce a hypoalgesic effect. These data are important as they have the potential to guide the selection of exercise parameters for future clinical trials examining the effectiveness of aerobic exercise to improve pain sensitivity. However, a broader approach examining other key outcomes, such as disability and health-related quality of life, will also be important. The randomised controlled trial by Ote Karaca et al. was the only trial to perform testing of PPTs and patient surveys for health-related quality of life measures before and after the intervention, with the SF-36 revealing that the aerobic exercise group benefited with improved general health perceptions and reduced role limitations because of physical problems compared with the control group [51].

Three observational studies examined whether subgroups of individuals with musculoskeletal pain differ in their pain sensitivity response to aerobic exercise. Vaegter et al. 2016 found that although individuals with high pain sensitivity experienced increased PPTs post exercise, there was a slight impairment of their exercise-induced hypoalgesia compared to those with low pain sensitivity [45]. In contrast, Fingleton et al. found that individuals with knee OA and abnormal conditioned pain modulation demonstrated increased pain sensitivity compared to those with normal conditioned pain modulation and healthy controls, and Vaegter et al. 2021 found that individuals who reported an increase in lower back or leg pain with walking had impaired exercise induced hypoalgesia compared those individuals with no increased pain with walking [45]. It is worth noting that while these subgroups have been designed as surrogates for an altered pain response at baseline, these studies defined them differently. These findings demonstrate that not all individuals with chronic pain have the same response to the prescribed aerobic exercise. We postulate that individuals

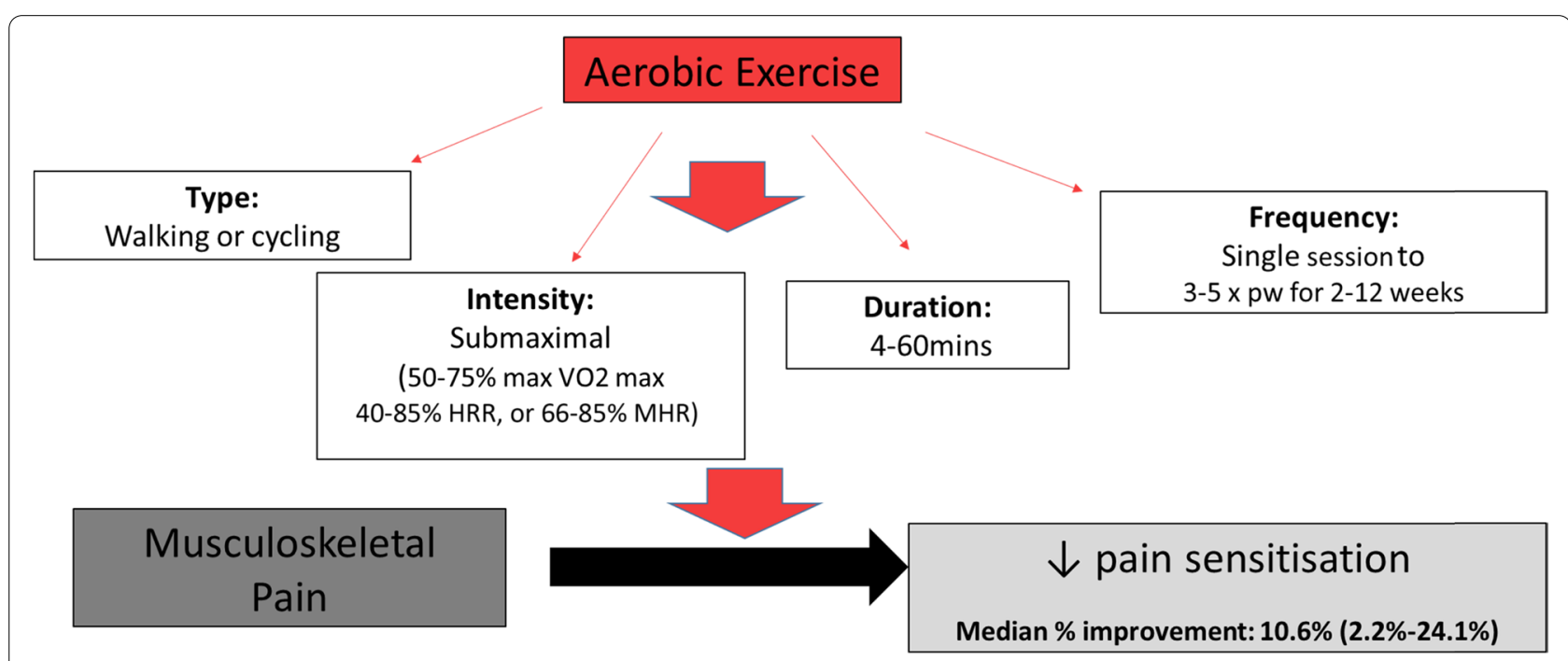

Fig. 2 Schematic diagram showing the effect of aerobic exercise on pain sensitisation for musculoskeletal pain. Note: colour print is not required 
with a higher pain sensitivity or abnormal conditioned pain modulation at baseline might be an inherently different subgroup of patients with chronic pain, and may exhibit similar responses to aerobic exercise as patients with fibromyalgia $[55,56]$. Thus, specific patient subgroups among those with chronic musculoskeletal pain need to be considered, and potentially each assessed and prescribed exercise in a specific way to ensure benefit from such an approach.

Several mechanisms have been proposed to explain how aerobic exercise may reduce pain sensitivity. While it has been suggested that aerobic exercise may release endogenous opioids and beta-endorphins that result in hypoalgesia, it has also been proposed that it may activate descending nociceptive inhibitory mechanisms that reduce pain sensitivity [24-26, 57, 58]. Moreover, the conditioned pain modulation theory hypothesizes that descending pain inhibitory responses are challenged during a painful conditioning stimulus via opioid and nonopioid pathways [58]. For instance, lower pain scores during exercise might be reported in the presence of placing a hand in ice water (the conditioning stimulus) [58]. The evidence from this review, which indicates that aerobic exercise reduces pain sensitisation in musculoskeletal pain, has the potential to have clinical implications. This is because, current clinical guidelines recommend the use of physical activity for musculoskeletal pain, but do not suggest a particular type of exercise, nor specifically recommend aerobic exercise. For instance, the NICE guidelines for non-specific low back pain recommend the provision of a group exercise program, but state an inability to recommend aerobic exercise alone due to the uncertainty around the effect size, clinical importance of the comparisons supporting aerobic exercise, as well as lack of an economic evaluation for an individual versus group intervention [59]. Thus, clinical trials examining the efficacy of aerobic exercise for specific patient subgroups, in particular those with pain sensitisation, and that involve large sample sizes and an economic evaluation, are urgently needed. These trials have the potential to inform clinical practice and treatment guidelines surrounding exercise recommendations for the future management of musculoskeletal pain, in particular, low back pain.

This review has several strengths and limitations. The key strengths included performing systematic searches of 6 electronic databases and reference lists of key systematic reviews, summarising and tabulating data from the included studies, performing a risk of bias assessment based on Cochrane methodology, and conducting a qualitative analysis, based on study design and quality. This review was limited by the modest number of heterogeneous studies, which prevented a meta-analysis being performed. However, we were able to report a decrease in pain sensitisation across all studies irrespective of study quality and a median improvement of $10 \%$ or more in pain sensitivity in studies which provided the appropriate data. While there is no established minimal clinically important difference (MCID) for pain sensitisation, there is evidence from a recent meta-analysis that patients who experience chronic pain have a significantly lower pressure pain threshold than healthy controls [60]. The pooled pressure pain threshold mean difference was reported as $1.17(95 \%$ confidence interval $=-1.45$ to -0.90$) \mathrm{kg} / \mathrm{cm}^{2}$, representing a $10 \%$ difference in PPTs between healthy individuals and those with chronic pain, and may indicate that a similar change of $10.6 \%$ in PPTs in individuals with chronic musculoskeletal pain following aerobic exercise is of clinical significance. We excluded nonEnglish studies, which may have introduced bias into the review. Although unlikely, it may be that the effect of aerobic exercise on pain sensitisation differs across populations. Moreover, while our risk of bias assessment based on Cochrane methodology reported that the studies were mainly of modest quality, this review will inform future research, in particular the need for high quality clinical trials, longer follow up periods, and a greater focus on global outcomes, including disability and quality of life.

\section{Conclusions}

This systematic review provides evidence to suggest that aerobic exercise reduces pain sensitisation in individuals with musculoskeletal pain. The findings suggest that aerobic exercise involving walking or cycling, performed at a submaximal intensity but with incremental increases, for a 4-60 min duration and for up to 12 weeks can produce a median (minimum, maximum) percentage improvement of $10.6 \%(2.2 \%, 24.1 \%)$ in pain sensitisation. These findings support the need for further work to determine whether the effect of aerobic exercise on pain sensitisation in individuals with musculoskeletal pain also translates to improved clinical outcomes.

Abbreviations

OA: osteoarthritis; PPT: pressure pain thresholds; TPT: thermal pain thresholds.

\section{Supplementary Information}

The online version contains supplementary material available at https://doi. org/10.1186/s12891-022-05047-9.

Additional file 1. 


\section{Acknowledgements}

We would like to acknowledge Mr Adam Clark for his assistance with referencing software.

\section{Authors' contributions}

The study was conceptualised by DU and FC. LR, DU and LT conceived the search strategy. LR ran the searches. LT and DU screened the studies and a consensus method was used to resolve any disagreements. LT and DU extracted the data and the ROB assessment was performed by JF and LT independently, with disagreements resolved by DU and SMH. LT and DU were major contributors in writing the manuscript. All authors read and approved the final manuscript.

\section{Authors' information}

Not applicable.

\section{Funding}

FMC, SMH and DU are recipients of a National Health and Medical Research Council (NHMRC) Investigator Grant (APP1 194829), NHMRC Early Career Fellowship (APP1142198) and NHMRC/Medical Research Future Fund (MRFF) Career Development Fellowship (Clinical Level 2 \#1142809) respectively. This research did not receive any specific grant from funding agencies in the public, commercial or non-for-profit sectors. Funding sources did not have any involvement in the study design; the collection, analysis and interpretation of data; the writing of the report; or the decision to submit the article for publication.

\section{Availability of data and materials}

All data generated or analysed during this study are included in this published article [and its supplementary information files].

\section{Declarations}

Ethics approval and consent to participate

Not applicable.

\section{Consent for publication}

Not applicable.

\section{Competing interests}

The authors declare no financial or non-financial competing interests.

\section{Author details}

'Department of Epidemiology and Preventive Medicine, School of Public Health and Preventive Medicine, Monash University, 553 St Kilda Rd, Melbourne, Victoria 3004, Australia. ${ }^{2}$ The lan Potter Library, The Alfred Hospital, Melbourne, Victoria 3004, Australia.

Received: 22 July 2021 Accepted: 17 January 2022

Published online: 03 February 2022

\section{References}

1. Briggs AM, Cross MJ, Hoy DG et al. Musculoskeletal health conditions represent a global threat to healthy aging: a report for the 2015 World Health Organization world report on ageing and health. Gerontologist 2016;56 Suppl 2(S243-55.

2. Briggs $A M$, Woolf $A D$, Dreinhofer $K$ et al. Reducing the global burden of musculoskeletal conditions. Bull World Health Organ 2018;96(5):366-8.

3. Disease GBD, Injury I, Prevalence C. Global, regional, and national incidence, prevalence, and years lived with disability for 328 diseases and injuries for 195 countries, 1990-2016: a systematic analysis for the Global Burden of Disease Study 2016. Lancet 2017;390(10100):1211-59.

4. Perrot S, Cohen M, Barke A et al. The IASP classification of chronic pain for ICD-11: chronic secondary musculoskeletal pain. Pain 2019;160(1):77-82.

5. Arendt-Nielsen L, Graven-Nielsen T. Translational musculoskeletal pain research. Best Pract Res Clin Rheumatol 2011;25(2):209-26.

6. International Association for the Study of Pain. IASP terminology Washington, D.C.: International Association for the Study of Pain; 2017
[Available from: https://www.iasp-pain.org/Education/Content.aspx? ItemNumber $=1698 \#$ Sensitization.

7. Lluch E, Torres R, Nijs J et al. Evidence for central sensitization in patients with osteoarthritis pain: a systematic literature review. Eur J Pain 2014;18(10):1367-75.

8. Coronado RA, Bialosky JE, Robinson ME et al. Pain sensitivity subgroups in individuals with spine pain: potential relevance to short-term clinical outcome. Phys Ther 2014;94(8):1111-22.

9. Fingleton C, Smart K, Moloney N et al. Pain sensitization in people with knee osteoarthritis: a systematic review and meta-analysis. Osteoarthritis Cartilage 2015;23(7):1043-56.

10. Smart KM, Blake C, Staines A et al. Self-reported pain severity, quality of life, disability, anxiety and depression in patients classified with 'nociceptive', 'peripheral neuropathic' and 'central sensitisation' pain. The discriminant validity of mechanisms-based classifications of low back (+/-leg) pain. Man Ther 2012;17(2):119-25.

11. Arendt-Nielsen L, Morlion B, Perrot S et al. Assessment and manifestation of central sensitisation across different chronic pain conditions. Eur J Pain 2018;22(2):216-41.

12. Nijs J, Malfliet A, Ickmans K et al. Treatment of central sensitization in patients with 'unexplained' chronic pain: an update. Expert Opin Pharmacother 2014;15(12):1671-83.

13. Chou R, Turner JA, Devine EB et al. The effectiveness and risks of longterm opioid therapy for chronic pain: a systematic review for a National Institutes of Health pathways to prevention workshop. Ann Intern Med 2015;162(4):276-86.

14. Goesling J, Henry MJ, Moser SE et al. Symptoms of depression are associated with opioid use regardless of pain severity and physical functioning among treatment-seeking patients with chronic pain. J Pain 2015;16(9):844-51.

15. Higgins C, Smith BH, Matthews K. Evidence of opioid-induced hyperalgesia in clinical populations after chronic opioid exposure: a systematic review and meta-analysis. Br J Anaesth 2019;122(6):e114-e26.

16. Riediger C, Schuster T, Barlinn K et al. Adverse effects of antidepressants for chronic pain: a systematic review and meta-analysis. Front Neurol 2017;8(307.

17. Rivat C, Ballantyne J. The dark side of opioids in pain management: basic science explains clinical observation. Pain Rep 2016;1(2):e570.

18. Registered Nurses' Association of Ontario (RNAO). Assessment and management of pain2013. Available from: https://rnao.ca/sites/rnaoca/files/AssessAndManagementOfPain2014.pdf.

19. Scottish Intercollegiate Guidelines Network (SIGN). Management of chronic pain2013. Available from: http://sign.ac.uk/guidelines/fulltext/ 136/index.html.

20. Hooten WM TR, Belgrade M, Gaul J, Goertz M, Haake B, Myers C, Noonan MP, Owens J, Saeger L, Schweim K SG, Walker N. Assessment and management of chronic pain2013. Available from: http://formsus.datas us.gov.br/novoimgarq/33266/6201476_353278.pdf.

21. University of Michigan Health System Chronic Pain Management Guideline Team. Managing chronic non-terminal pain in adults including prescribing controlled substances2011 20th March 2021. Available from: http://www.med.umich.edu/1 info/FHP/practiceguides/pain/pain.pdf.

22. Busch AJ, Webber SC, Brachaniec $M$ et al. Exercise therapy for fibromyalgia. Curr Pain Headache Rep 2011;15(5):358-67.

23. Patel H, Alkhawam H, Madanieh R et al. Aerobic vs anaerobic exercise training effects on the cardiovascular system. World J Cardiol 2017:9(2):134-8.

24. Dietrich A, McDaniel WF. Endocannabinoids and exercise. $\mathrm{Br} J$ Sports Med 2004;38(5):536-41.

25. Pertwee RG. Cannabinoid receptors and pain. Prog Neurobiol 2001;63(5):569-611.

26. Thoren P, Floras JS, Hoffmann P et al. Endorphins and exercise: physiological mechanisms and clinical implications. Med Sci Sports Exerc 1990;22(4):417-28.

27. Naugle KM, Fillingim RB, Riley JL, III. A meta-analytic review of the hypoalgesic effects of exercise. J Pain 2012;13(12):1139-50.

28. Larun L, Brurberg KG, Odgaard-Jensen J et al. Exercise therapy for chronic fatigue syndrome. Cochrane Database Syst Rev 2019;10(CD003200. 
29. Nijs J, Kosek E, Van Oosterwijck J et al. Dysfunctional endogenous analgesia during exercise in patients with chronic pain: to exercise or not to exercise? Pain Physician 2012;15(3 Suppl):ES205-13.

30. Hurkmans E, van der Giesen FJ, Vliet Vlieland TP et al. Dynamic exercise programs (aerobic capacity and/or muscle strength training) in patients with rheumatoid arthritis. Cochrane Database Syst Rev 2009;4):CD006853.

31. Newcomb LW, Koltyn KF, Morgan WP et al. Influence of preferred versus prescribed exercise on pain in fibromyalgia. Med Sci Sports Exerc 2011:43(6):1106-13.

32. Vierck CJ, Jr., Staud R, Price DD et al. The effect of maximal exercise on temporal summation of second pain (windup) in patients with fibromyalgia syndrome. J Pain 2001;2(6):334-44.

33. Meeus M, Roussel NA, Truijen S et al. Reduced pressure pain thresholds in response to exercise in chronic fatigue syndrome but not in chronic low back pain: an experimental study. J Rehabil Med 2010;42(9):884-90.

34. Page M, McKenzie J, Bossuyt P et al. The PRISMA 2020 statement: An updated guideline for reporting systematic reviews. J Clin Epidemiol 2021;134(178-89.

35. Balaguier R, Madeleine P, Vuillerme $\mathrm{N}$. Is one trial sufficient to obtain excellent pressure pain threshold reliability in the low back of asymptomatic Individuals? A test-retest study. PLoS One 2016;11(8):e0160866.

36. Maquet D, Croisier JL, Demoulin C et al. Pressure pain thresholds of tender point sites in patients with fibromyalgia and in healthy controls. Eur J Pain 2004;8(2):111-7.

37. Hoffman MD, Shepanski MA, Mackenzie SP et al. Experimentally induced pain perception is acutely reduced by aerobic exercise in people with chronic low back pain. J Rehabil Res Dev 2005:42(2):183-90.

38. World Health Organisation. Musculoskeletal conditions: World Health Organisation; 2019 [Available from: https://www.who.int/news-room/ fact-sheets/detail/musculoskeletal-conditions.

39. Fredriksson $L$, Alstergren $P, K o p p ~ S$. Absolute and relative facial pressurepain thresholds in healthy individuals. J Orofac Pain 2000;14(2):98-104.

40. Laursen BS, Bajaj P, Olesen AS et al. Health related quality of life and quantitative pain measurement in females with chronic non-malignant pain. Eur J Pain 2005:9(3):267-75.

41. Fingleton C, Smart KM, Doody CM. Exercise-induced hypoalgesia in people with knee osteoarthritis with normal and abnormal conditioned pain modulation. Clin J Pain 2017:33(5):395-404.

42. Higgins J, Altman D, Jonathon S. Assessing risk of bias in included studies. In: Higgins J, Green S, editors. Cochrane handbook for systematic reviews of interventions. 5.1.0 (updated March 2011): The Cochrane Collaboration, 2011.; 2011. p. Available from www.handbook.cochrane.org.

43. Higgins J, Altman D, Gøtzsche P et al. The Cochrane Collaboration's tool for assessing risk of bias in randomised trials. BMJ 2011;343(d5928.

44. Barbateskovic M, Koster T, Eck R et al. A new tool to assess Clinical Diversity In Meta-analyses (CDIM) of interventions. J Clin Epidemiol 2021;135(29-41.

45. Vaegter HB, Handberg G, Graven-Nielsen T. Hypoalgesia after exercise and the cold pressor test is reduced in chronic musculoskeletal pain patients with high pain sensitivity. Clin J Pain 2016;32(1):58-69.

46. Vaegter HB, Madsen AB, Handberg $G$ et al. Kinesiophobia is associated with pain intensity but not pain sensitivity before and after exercise: an explorative analysis. Physiotherapy 2018;104(2):187-93.

47. Vaegter H, Petersen K, Sjodsholm L et al. Impaired exercise-induced hypoalgesia in individuals reporting an increase in low back pain during acute exercise. Eur J Pain 2021;25(5):1053-63.

48. Sitges C, Velasco-Roldán O, Crespí J et al. Acute effects of a brief physical exercise intervention on somatosensory perception, lumbar strength, and flexibility in patients with nonspecific chronic low-back pain. J Pain Res 2021;14(487-500.

49. Kocur P, Pospieszna B, Choszczewski D et al. The effects of Nordic Walking training on selected upper-body muscle groups in female-office workers: a randomized trial. Work 2017;56(2):277-83.

50. Nielsen PK, Andersen LL, Olsen HB et al. Effect of physical training on pain sensitivity and trapezius muscle morphology. Muscle and Nerve 2010;41(6):836-44.

51. Ote Karaca S, Demirsoy N, Gunendi Z. Effects of aerobic exercise on pain sensitivity, heart rate recovery, and health-related quality of life in patients with chronic musculoskeletal pain. International journal of rehabilitation research 2017; Internationale Zeitschrift fur Rehabilitationsforschung. Revue internationale de recherches de readaptation. 40(2):164-70.
52. Bruehl S, Burns J, Koltyn K et al. Are endogenous opioid mechanisms involved in the effects of aerobic exercise training on chronic low back pain? A randomized controlled trial. Pain 2020;161(12):2887-97.

53. National Health Service. Prescriptions dispensed in the community statistics for England, 2005-2015. United Kingdom: Health and Social Care Information Centre (HSCIC), National Health Service; 2018.

54. Wong J, Motulsky A, Eguale T et al. Treatment indications for antidepressants prescribed in primary care in Quebec, Canada, 2006-2015. JAMA 2016;315(20):2230-2

55. O'Brien AT, Deitos A, Trinanes Pego Y et al. Defective endogenous pain modulation in fibromyalgia: a meta-analysis of temporal summation and conditioned pain modulation paradigms. J Pain 2018;19(8):819-36.

56. Richard JY, Hurley RA, Taber KH. Fibromyalgia: centralized pain processing and neuroimaging. J Neuropsychiatry Clin Neurosci 31(3):A6-187.

57. Kjaer M, Secher NH, Bach FW et al. Hormonal, metabolic, and cardiovascular responses to static exercise in humans: influence of epidural anesthesia. Am J Physiol 1991;261(2 Pt 1):E214-20.

58. Smith BE, Hendrick $P$, Bateman $M$ et al. Musculoskeletal pain and exercisechallenging existing paradigms and introducing new. Br J Sports Med 2019;53(14):907-12.

59. National Institute for Health and Care Excellence (NICE). Low back pain and sciatica in over 16s: assessment and management. London, UK2020.

60. Amiri $M$, Alavinia $M$, Singh $M$, et al. Pressure pain threshold in patients with chronic pain: A systematic review and meta-analysis. Am J Phys Med Rehabil. 2021;100(7):656-74.

\section{Publisher's Note}

Springer Nature remains neutral with regard to jurisdictional claims in published maps and institutional affiliations.

Ready to submit your research? Choose BMC and benefit from

- fast, convenient online submission

- thorough peer review by experienced researchers in your field

- rapid publication on acceptance

- support for research data, including large and complex data types

- gold Open Access which fosters wider collaboration and increased citations

- maximum visibility for your research: over $100 \mathrm{M}$ website views per year

At BMC, research is always in progress.

Learn more biomedcentral.com/submissions 